\section{BMJ}

Open

Gastroenterology

\title{
Impact of comorbidities on anti-TNFa response and relapse in patients with inflammatory bowel disease: the VERNE study
}

\author{
Ignacio Marin-Jimenez (D) ,1,2 Guillermo Bastida, ${ }^{3}$ Ana Forés, ${ }^{4}$ \\ Esther Garcia-Planella, ${ }^{5}$ Federico Argüelles-Arias, ${ }^{6}$ Pilar Sarasa, ${ }^{7}$ Ignacio Tagarro, ${ }^{7}$ \\ Alonso Fernández-Nistal, ${ }^{7}$ Carmen Montoto, ${ }^{7}$ Mariam Aguas, ${ }^{3}$ \\ Javier Santos-Fernández, ${ }^{8}$ Marta Maia Bosca-Watts, ${ }^{9}$ Rocio Ferreiro, ${ }^{10}$ \\ Olga Merino, ${ }^{11}$ Xavier Aldeguer, ${ }^{12}$ Xavier Cortés, ${ }^{13}$ Beatriz Sicilia, ${ }^{14}$ \\ Francisco Mesonero, ${ }^{15}$ Manuel Barreiro-de Acosta ${ }^{16}$
}

To cite: Marin-Jimenez I, Bastida G, Forés A, et al. Impact of comorbidities on antiTNF $\alpha$ response and relapse in patients with inflammatory bowel disease: the VERNE study. BMJ Open Gastro 2020;7:e000351. doi:10.1136/ bmjgast-2019-000351

Received 24 October 2019 Revised 13 January 2020 Accepted 19 January 2020
Check for updates

(C) Author(s) (or their employer(s)) 2020. Re-use permitted under CC BY-NC. No commercial re-use. See rights and permissions. Published by BMJ.

For numbered affiliations see end of article.

Correspondence to Dr Ignacio Marin-Jimenez; drnachomarin@hotmail.com

\section{ABSTRACT}

Objective To evaluate the impact of comorbidities and extraintestinal manifestations of inflammatory bowel disease on the response of patients with inflammatory bowel disease to antitumour necrosis factor alpha (anti$\mathrm{TNF} \alpha$ ) therapy.

Design Data from 310 patients (194 with Crohn's disease and 116 with ulcerative colitis) treated consecutively with the first anti-TNF $\alpha$ in 24 Spanish hospitals were retrospectively analysed. Univariate and multivariate logistic regression analyses were performed to assess the associations between inflammatory bowel disease comorbidities and extraintestinal manifestations with anti-TNF $\alpha$ treatment outcomes. Key clinical features, such as type of inflammatory bowel disease and concomitant treatments, were included as fixed factors in the model.

Results Multivariate logistic regression analyses (OR, $95 \% \mathrm{Cl}$ ) showed that chronic obstructive pulmonary disease $(2.67,1.33$ to 5.35$)$ and hepato-pancreato-biliary diseases $(1.87,1.48$ to 2.36$)$ were significantly associated with primary non-response to anti-TNF $\alpha$, as was the use of corticosteroids and the type of inflammatory bowel disease (ulcerative colitis vs Crohn's disease). It was also found that myocardial infarction (3.30, 1.48 to 7.35$)$ and skin disease $(2.73,1.42$ to 5.25$)$ were significantly associated with loss of response, along with the use of corticosteroids and the type of inflammatory bowel disease (ulcerative colitis vs Crohn's disease).

Conclusions Our results suggest that the presence of some comorbidities in patients with inflammatory bowel disease, such as chronic obstructive pulmonary disease and myocardial infarction, and of certain extraintestinal manifestations of inflammatory bowel disease, such as hepato-pancreato-biliary conditions and skin diseases, appear to be related to failure to anti-TNF $\alpha$ treatment. Therefore, their presence should be considered when choosing a treatment.

Trial registration number NCT02861118.
Summary box

What is already known about this subject?

- Different real-life studies have investigated factors that might predict response to antitumour necrosis factor alpha (anti-TNF $\alpha$ ) drugs in patients with inflammatory bowel disease (IBD); however, none has specifically addressed the impact of comorbidities and extraintestinal manifestation (EIM) profile on the response to these therapies.

What are the new findings?

- To the best of our knowledge, this is the first study to assess the association between comorbidities and EIM profile of patients with IBD and the response to the first treatment with an anti-TNF $\alpha$.

How might it impact on clinical practice in the foreseeable future?

- These results may be useful in selecting those patients who most likely can benefit from anti-TNFo therapies.

- Specifically, the results suggest that chronic obstructive pulmonary disease, myocardial infarction, hepato-pancreato-biliary conditions and skin diseases may have a negative influence on anti-TNF $\alpha$ treatment outcomes.

\section{INTRODUCTION}

Inflammatory bowel disease (IBD) describes a group of chronic, progressive disorders that predominantly affect the bowel. Although the pathophysiology is not fully known, it seems that environmental factors and an abnormal immune response to enteric microbes play a role in individuals with a genetic predisposition. ${ }^{1}$ Crohn's disease (CD) and ulcerative colitis (UC) are the two main forms of IBD. The disease often has an onset during young 
adulthood and a course characterised by remission and relapse phases. ${ }^{2}$ The progressive organ damage results in a negative impact on the patient's health-related quality of life (HRQoL) and in a major economic burden for both society and health services. ${ }^{3-7}$

The Spanish incidence of IBD seems to have increased in recent years, similarly to other countries, ${ }^{89}$ and ranges between 8 and 11 cases per 100000 inhabitants per year. $^{10-12}$

IBD has been frequently associated with comorbidities that, although not having a direct relationship with bowel inflammation, can modify the course and management outcomes of the disease. ${ }^{13}$ The prevalence of comorbidities in patients with IBD ranges between $30 \%$ and $70 \%{ }^{14-16}$ The presence of comorbidities in patients with IBD has been shown to negatively affect patients' HRQoL, ${ }^{16}$ and importantly to prolong their length of hospital stay and increase the risk of postsurgical mortality. Furthermore, immune-mediated inflammatory disorders associated with IBD have been proven to incur higher healthcare costs. ${ }^{14}$

In addition, up to $50 \%$ of patients with IBD may develop extraintestinal manifestations (EIMs) of the disease, ${ }^{17}$ involving multiple organ systems throughout the body, ${ }^{17}{ }^{18}$ and sometimes being even more debilitating than the intestinal disease itself. ${ }^{19}$

In recent decades, the objective of IBD treatment has evolved from a symptomatic relief to symptomatic and endoscopic deep remission. ${ }^{20}{ }^{21}$ Conventional treatment with corticosteroids and immunosuppressants has not been able to reduce the complications of the disease or modify its course. ${ }^{22-24}$ Over the last two decades, biologic treatments have been successfully used in patients with moderate to severe CD and UC who failed to respond to corticosteroids. ${ }^{25}$ Early introduction of biologic agents in patients with more serious disease is probably the most widely accepted management strategy. ${ }^{22-25}$ Antitumour necrosis factor alpha (anti-TNF $\alpha$ ) monoclonal antibodies, such as infliximab and adalimumab, have been rather effective in inducing and maintaining mucosal healing and reducing surgery and hospitalisation rates for over 15 years. Nonetheless, since anti-TNF $\alpha$ therapy failures are not uncommon, ${ }^{26}{ }^{27}$ many studies have investigated patient-related, disease-related and treatment-related factors predicting response to anti-TNF $\alpha$ agents in IBD. They have found associations among the response to anti-TNF $\alpha$ medications and disease duration, biomarker levels, genetic polymorphisms and immunopharmacological factors. ${ }^{27-30}$ However, there have not been studies conducted to determine the impact of comorbidities on anti-TNF $\alpha$ treatment response.

The primary objective of this study was to evaluate the impact of the comorbidity profile of patients with IBD on treatment response to the first anti-TNF $\alpha$ therapy. Secondarily, the impact of the EIM profile in patients with IBD on treatment response to the first anti-TNF $\alpha$ was assessed, the percentage of patients with IBD exhibiting comorbidities was described, and the comorbidity profile at each level of IBD severity was determined.

\section{METHODS}

\section{Study population}

This study was a retrospective, non-interventional, observational, multicentre study involving 24 gastroenterology sites from Spain. The study included patients diagnosed with UC or CD who started their first anti-TNF $\alpha$ between June 2011 and June 2013. To minimise selection bias, patients were recruited consecutively.

Patients had to be diagnosed with UC or CD according to the 'World Gastroenterology Organization Practice Guidelines for the Diagnosis and Management of IBD in 2010 ${ }^{31}$ All patients must have been prescribed anti$\mathrm{TNF} \alpha$ treatment according to daily clinical practice and have given written informed consent.

Investigators collected data from medical charts, including sociodemographic information (age, gender, race, level of education, smoking habits and alcohol intake) and clinical information (concomitant diseases, EIM of IBD, date of diagnosis, previous treatments, current treatments, disease activity when starting treatment with anti-TNF $\alpha$ ). When data were not properly recorded in the medical charts, particularly demographic information, they were obtained directly from patients during a routine visit.

\section{Clinical outcome evaluation}

Disease activity at the beginning of the reference period and at the study visit was assessed through the following variables: (1) for patients with UC, the Partial Mayo Score $(\mathrm{PMS})^{32}$ for general disease activity, disease anatomical extent, and endoscopy findings; (2) for patients with $\mathrm{CD}$, the Harvey-Bradshaw Index (HBI) ${ }^{33}$ for general disease activity, disease behaviour, and disease location; and (3) for patients with UC and CD, stool frequency, rectal bleeding, urgency, nocturnal stools, need for antidiarrhoeal drugs, constipation, abdominal tenderness, abdominal pain or cramping, anorexia, nausea, vomiting, fever, appetite loss, weight loss, fatigue, night sweats, stunted growth, primary amenorrhoea, general well-being, physician's global assessment, and other complications.

The reference period of the study was defined as the interval between the start of anti-TNF $\alpha$ until either the study visit or lack or loss of treatment response or until treatment discontinuation. To assess the main outcome, non-responders were defined as those patients not achieving either a reduction in HBI of at least two points from baseline for $\mathrm{CD},{ }^{32}$ or a decrease in PMS of at least two points for UC. ${ }^{33}$ In the cases where these indexes were not available, clinical response was evaluated according to physician criteria as recorded in medical charts. In both cases, response was assessed after induction treatment ( 10 weeks after starting anti-TNF $\alpha$ ) and after maintenance treatment (at least 6 months after starting 


\begin{tabular}{ll}
\hline Table 1 Charlson index & \\
\hline Comorbidity & Weighted index \\
\hline Myocardial infarction & 1 \\
Congestive heart failure & 1 \\
\hline Peripheral vascular disease & 1 \\
\hline Cerebrovascular disease & 1 \\
\hline Dementia & 1 \\
\hline Chronic obstructive pulmonary & 1 \\
disease & \\
\hline Connective tissue disease & 1 \\
\hline Peptic ulcer disease & 1 \\
\hline Diabetes mellitus & 1 (mild), 2 (moderate to \\
& severe) \\
Chronic renal disease & 2 (moderate to severe) \\
\hline Hemiplegia & 2 \\
Leukaemia & 2 \\
Lymphoma & 2 \\
\hline Solid tumour & 2 (tumour), 6 (metastatic) \\
Liver disease & 1 (mild), 3 (moderate to \\
\hline AlDS & severe) \\
\hline
\end{tabular}

anti-TNF $\alpha$ ). Primary non-response (PNR) was defined as lack of response after induction treatment (weeks 0-10). Loss of response (LOR) was defined as loss of the effect of the drug along the follow-up in a patient with an initial response. ${ }^{34}$ Reasons to stop the drug were classified as PNR, LOR, side effect, remission or other. ${ }^{34}$

IBD comorbidities in patients with UC and CD (defined as the coexistence of another medical condition alongside IBD that did not imply causation) at the time of diagnosis and at the study visit were listed according to the Charlson index ${ }^{35}$ (table 1). EIMs in patients with UC and CD were chosen from a list from the European Crohn's and Colitis Organisation guidelines. ${ }^{18}$

\section{Statistical analysis}

With an estimated $50 \%$ of non-responders to the first anti$\mathrm{TNF} \alpha$ treatment and considering a minimum sample of 10 patients who presented with the event of interest per independent variable included in the logistic regression model and a maximum of 10 independent variables per model, a sample of 200 patients with UC and 200 patients with $\mathrm{CD}$ were estimated.

All patients participating in the study who fulfilled the inclusion criteria and did not have a major deviation from the study protocol were included in the final analysis. Continuous variables were described as the number of patients with valid/missing observations, mean, and SD, or the median and IQR if required. Categorical variables were described by absolute and relative frequencies.

Pairwise comparisons were performed by t-tests, MannWhitney tests, analyses of variance or Kruskal-Wallis tests when comparing quantitative variables between study groups or categories and categorical variables, and with $\chi^{2}$ tests or Fisher's exact tests when comparing categorical variables.

To analyse the factors associated with PNR or LOR, a univariate analysis was performed including the sociodemographic variables, comorbidities, EIM, and other clinical variables as factors. Afterwards, a stepwise (backward and forward) multiple logistic regression analysis was performed using as the dependent variable either PNR or LOR and as independent variables those considered potential factors in the univariate analysis $(\mathrm{p}<0.100)$. In addition, given the potential relevance of the type of IBD (CD or UC) and of concomitant treatments on the response to anti-TNF $\alpha$ medications, the type of IBD and the concomitant administration of immunosuppressants and corticosteroids were included as fixed factors in the models.

An additional analysis to evaluate the impact of the EIM profile on treatment response to the first anti-TNF $\alpha$ therapy was conducted. It was based on two stepwise multiple logistic regression analyses with PNR or LOR as dependent variables and all EIMs (arthropathy and arthritis, metabolic bone disease, eye disease, oral, aural and nasal disease, skin disease, hepato-pancreato-biliary disease, neurological disease, and cardiovascular, pulmonary and genitourinary manifestations) as independent variables. Again, type of IBD, treatment with immunosuppressants, and treatment with corticosteroids were included as fixed factors in the models.

Additionally, we analysed the associations among comorbidities and IBD severity at baseline. Patients were categorised as having a severe disease if HBI was $>16$ or PMS was 8-9. We performed a univariate analysis considering severity as the dependent variable and type of IBD and comorbidities as independent variables. Afterwards, a stepwise multiple logistic regression analysis was performed using non-severe disease as the dependent variable and as independent variables those that were significant at $\mathrm{p}<0.10$ in the univariate analysis.

All data analyses were performed using IBM SPSS Statistics V.22.0 Statistical Package for Windows.

\section{RESULTS}

\section{Patient characteristics}

A total of 357 patients with IBD were included in this study. Forty-seven patients were eventually excluded from the analysis due to screening failure. Of the 310 analysed patients, 194 were diagnosed with $\mathrm{CD}$ and 116 were diagnosed with UC. Subjects' characteristics are shown in table 2. The mean age (SD) at the time of anti-TNFo treatment initiation was 38.8 (12.7) years for $\mathrm{CD}$ and 41.8 (13.3) years for UC. Men comprised $53.5 \%$ of the whole analysed population. There was a higher prevalence of non-smokers among patients with UC (60.3\%) compared with patients with CD (44.3\%). Likewise, there was a higher prevalence of ex-smokers in the UC subpopulation $(32.0 \%)$ compared with patients with $\mathrm{CD}$ 
Table 2 Demographics and clinical characteristics

\begin{tabular}{|c|c|c|c|c|c|c|}
\hline & CD & & UC & & Total & \\
\hline & $n$ & $\%$ & $n$ & $\%$ & $\mathbf{n}$ & $\%$ \\
\hline Gender & & & & & & \\
\hline Male & 103 & 53.10 & 63 & 54.30 & 166 & 53.50 \\
\hline Female & 90 & 46.40 & 47 & 40.50 & 137 & 44.20 \\
\hline Not available & 1 & 0.50 & 6 & 5.20 & 7 & 2.30 \\
\hline Total & 194 & 100 & 116 & 100 & 310 & 100 \\
\hline Ethnicity & & & & & & \\
\hline Caucasian & 189 & 97.50 & 108 & 93 & 297 & 95.80 \\
\hline Latin & 3 & 1.50 & 1 & 0.90 & 4 & 1.30 \\
\hline Gypsy & 1 & 0.50 & 0 & 0 & 1 & 0.30 \\
\hline Arab & 0 & 0 & 1 & 0.90 & 1 & 0.30 \\
\hline Not available & 1 & 0.50 & 6 & 5.20 & 7 & 2.30 \\
\hline Total & 194 & 100 & 116 & 100 & 310 & 100 \\
\hline Working status & & & & & & \\
\hline Employed by self & 115 & 59.30 & 51 & 44 & 166 & 53.50 \\
\hline Self-employed & 15 & 7.70 & 17 & 14.50 & 32 & 10.30 \\
\hline Retired & 16 & 8.20 & 16 & 13.80 & 32 & 10.30 \\
\hline Housework & 12 & 6.20 & 11 & 9.50 & 23 & 7.50 \\
\hline Unemployed & 17 & 8.80 & 4 & 3.40 & 21 & 6.80 \\
\hline Student & 6 & 3.10 & 6 & 5.20 & 12 & 3.90 \\
\hline Permanently unable to work & 6 & 3.10 & 3 & 2.60 & 9 & 2.90 \\
\hline Temporarily unable to work & 4 & 2.10 & 1 & 0.90 & 5 & 1.60 \\
\hline Other & 1 & 0.50 & 1 & 0.90 & 2 & 0.60 \\
\hline Not available & 2 & 1 & 6 & 5.20 & 8 & 2.60 \\
\hline Total & 194 & 100 & 116 & 100 & 310 & 100 \\
\hline Level of education & & & & & & \\
\hline Secondary education & 101 & 52.10 & 46 & 39.60 & 147 & 48.50 \\
\hline Primary education & 45 & 23.20 & 30 & 25.90 & 75 & 24.50 \\
\hline University education & 42 & 21.60 & 32 & 27.60 & 74 & 24.30 \\
\hline Uneducated & 4 & 2.10 & 2 & 1.70 & 6 & 1.90 \\
\hline Not available & 2 & 1 & 6 & 5.20 & 8 & 2.70 \\
\hline Total & 194 & 100 & 116 & 100 & 310 & 100 \\
\hline Smoking habits & & & & & & \\
\hline Non-smoker & 86 & 44.30 & 70 & 60.30 & 156 & 50.30 \\
\hline Ex-smoker & 52 & 26.80 & 37 & 32 & 89 & 28.70 \\
\hline Smoker & 55 & 28.40 & 7 & 6 & 62 & 20 \\
\hline Not available & 1 & 0.50 & 2 & 1.70 & 3 & 1 \\
\hline Total & 194 & 100 & 116 & 100 & 310 & 100 \\
\hline Alcohol abuse & & & & & & \\
\hline No & 189 & 97.40 & 114 & 98.30 & 303 & 97.70 \\
\hline Yes & 4 & 2.10 & 0 & 0 & 4 & 1.30 \\
\hline Not available & 1 & 0.50 & 2 & 1.70 & 3 & 1 \\
\hline Total & 194 & 100 & 116 & 100 & 310 & 100 \\
\hline
\end{tabular}

Continued 
Table 2 Continued

\begin{tabular}{|c|c|c|c|c|c|c|}
\hline & $\mathbf{n}$ & Mean & Median & SD & $\begin{array}{l}\text { 25th } \\
\text { percentile }\end{array}$ & $\begin{array}{l}\text { 75th } \\
\text { percentile }\end{array}$ \\
\hline \multicolumn{7}{|c|}{ Age at signed informed consent (years) } \\
\hline $\mathrm{CD}$ & 194 & 43.8 & 43 & 12.8 & 34.0 & 51.3 \\
\hline UC & 116 & 46.8 & 46 & 13.3 & 38.0 & 57.8 \\
\hline Total & 310 & 44.9 & 44 & 13 & 36.0 & 53.3 \\
\hline \multicolumn{7}{|c|}{ Time from diagnosis of IBD to anti-TNF $\alpha$ treatment initiation (months) } \\
\hline CD & $193^{*}$ & 89.5 & 45.5 & 97.8 & 11.6 & 156 \\
\hline UC & $115^{*}$ & 77.6 & 43.8 & 82.1 & 10.8 & 143.8 \\
\hline Total & 308 & 85.0 & 45.5 & 92.3 & 11.1 & 150.2 \\
\hline \multicolumn{7}{|c|}{ Follow-up time from anti-TNF $\alpha$ treatment initiation (months) } \\
\hline CD & 92 & 59.5 & 59.8 & 7.9 & 53.2 & 65.8 \\
\hline UC & 65 & 59.5 & 59.8 & 7.5 & 53.4 & 65.1 \\
\hline Total & 157 & 59.5 & 59.8 & 7.7 & 53.3 & 65.6 \\
\hline
\end{tabular}

${ }^{*}$ Not available: 1 patient.

anti-TNF $\alpha$, antitumour necrosis factor alpha; CD, Crohn's disease; IBD, inflammatory bowel disease; UC, ulcerative colitis.

(26.8\%). The median (IQR) time from diagnosis of IBD to anti-TNF $\alpha$ treatment initiation was 45.5 (11.6-156.0) and 43.8 (10.8-143.8) months for CD and UC, respectively. Eighty-two per cent of patients reported having been previously treated with corticosteroids, and $33.3 \%$ were still receiving concomitant corticosteroid therapy during the maintenance phase. In addition, $78.4 \%$ of the study patients had been previously treated with immunosuppressants, and $65 \%$ were still under immunosuppressant therapy during the maintenance phase.

\section{Comorbidities and EIMs}

The Charlson comorbidity index mean values for CD were 0.21 (SD: 0.57), and 0.27 (SD: 0.72) for patients with UC. The prevalence of all individual comorbidities identified was below 6\% (figure 1). Diabetes mellitus

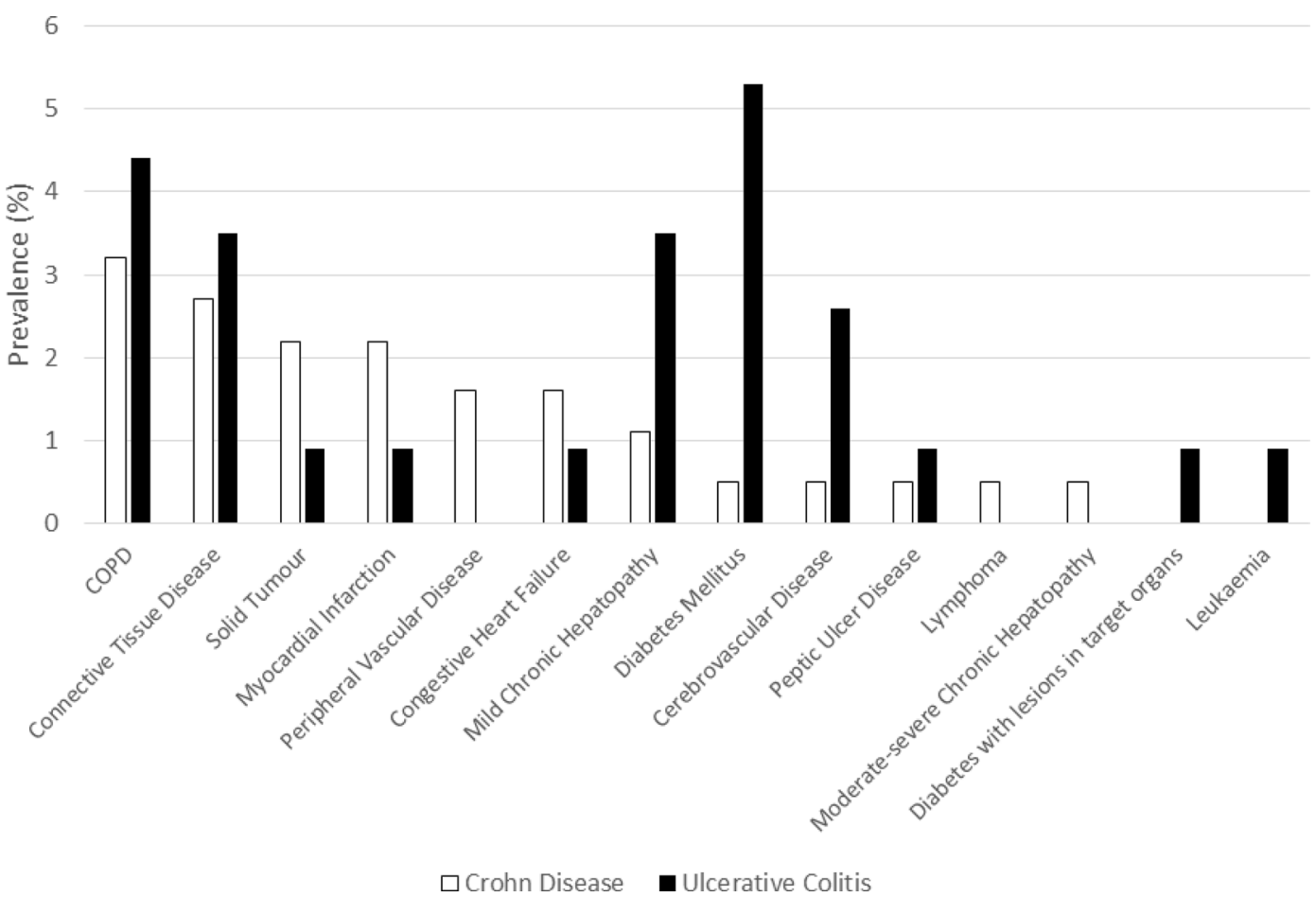

Figure 1 Prevalence of comorbidities. The following comorbidities presented a prevalence of 0\%: dementia, hemiplegia, moderate-severe chronic kidney disease, solid tumours with metastases, and AIDS. COPD, chronic obstructive pulmonary disease. 
Table 3 Prevalence of extraintestinal manifestations of IBD

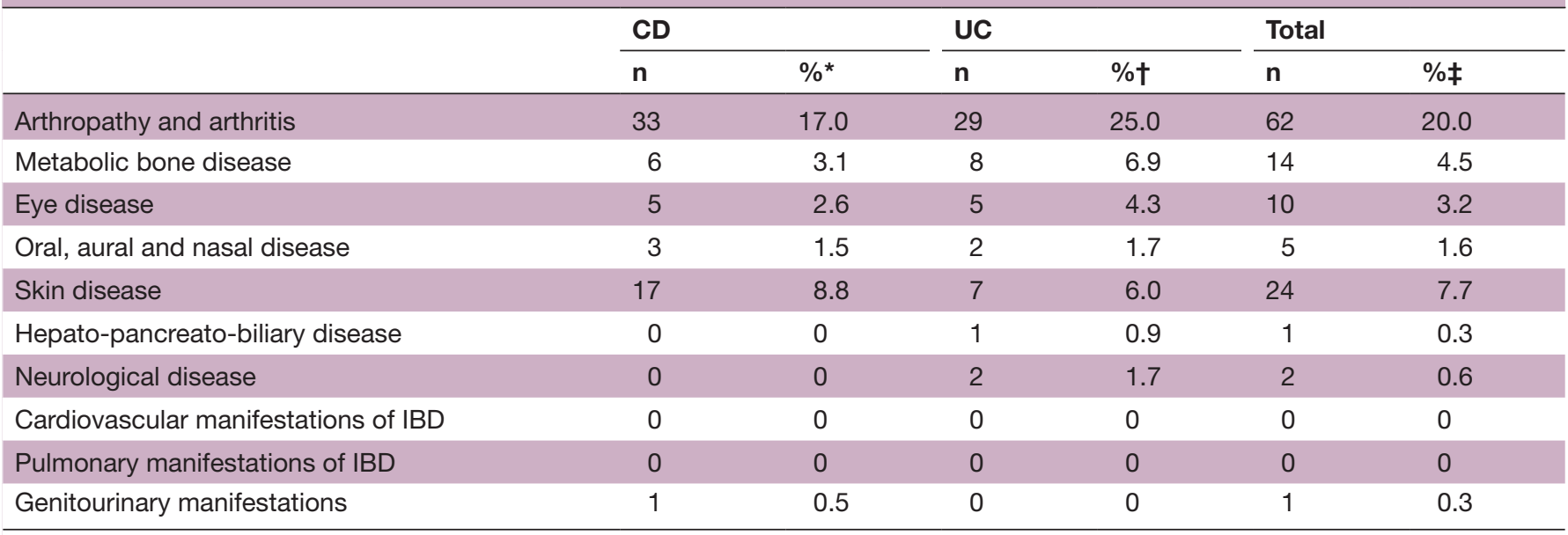

*Percentages calculated over 194 patients with CD.

†Percentages calculated over 116 patients with UC.

†Percentages calculated over 310 patients.

$\mathrm{CD}$, Crohn's disease; IBD, inflammatory bowel disease; UC, ulcerative colitis.

(5.3\%) was the most prevalent comorbidity for UC, and chronic obstructive pulmonary disease (COPD) was the most prevalent among patients with CD $(3.2 \%)$.

The prevalence of EIMs was higher in the UC subpopulation $(32.8 \%$ vs $26.3 \%)$. The most frequent EIMs (table 3 ) were arthropathy and arthritis $(17 \%$ for $\mathrm{CD}$ and $25 \%$ for $\mathrm{UC})$, skin disease ( $8.8 \%$ for $\mathrm{CD}$ and $6 \%$ for $\mathrm{UC})$, and metabolic bone disease ( $6.9 \%$ for UC).

\section{Factors associated with PNR and LOR to anti-TNF $\alpha$}

In the univariate analysis of comorbidities, COPD and solid tumours were identified as potential factors $(\mathrm{p}<0.10)$ associated with PNR. Multivariate logistic regression analysis showed that COPD (OR 2.67, 95\% CI 1.33 to 5.35) was significantly associated with PNR to anti-TNF $\alpha$, as was the use of corticosteroids and the type of IBD (UC vs CD) (table 4).

In the univariate analysis, myocardial infarction and skin disease were identified as potential factors $(\mathrm{p}<0.10)$ of LOR to anti-TNF $\alpha$ during the maintenance phase. Multivariate logistic regression analysis confirmed the association of myocardial infarction (OR 3.30, 95\% CI 1.48 to 7.35 ) and skin disease (OR 2.73, 95\% CI 1.42 to $5.25)$, along with the use of corticosteroids and the type of IBD (UC vs CD) (table 4), with LOR.

The additional analysis to evaluate the impact of the EIM profile in patients with IBD on treatment response to the first anti-TNF $\alpha$ therapy showed that the only EIM significantly associated with PNR was hepato-pancreatobiliary disease (OR 1.87, 95\% CI 1.48 to 2.36), and the only EIM associated with LOR was skin disease (OR 2.58, $95 \%$ CI 1.98 to 3.35 ) (table 4 ).

\section{Factors associated with IBD severity}

In the univariate analysis, IBD type, cerebrovascular disease, leukaemia, diabetes mellitus, and diabetes with injuries to target organs were potential factors associated with IBD severity $(\mathrm{p}<0.1)$. UC, compared with $\mathrm{CD}$, was significantly associated with more severe disease severity (OR $18.26,95 \%$ CI 2.32 to 143.62 ).

\section{DISCUSSION}

Although several studies have investigated the clinical and demographic factors that might predict PNR or LOR to anti-TNF $\alpha$ drugs in IBD, to the best of our knowledge, this is the first study to assess the association between the comorbidity profile in patients with IBD and PNR or LOR to anti-TNF $\alpha$ drugs.

The advent of anti-TNF $\alpha$ drugs supposed a substantial improvement in the management of IBD. However, up to $30 \%$ of patients do not respond to anti-TNF $\alpha$ therapy during the treatment induction phase (PNR), and $13 \%-20 \%$ lose the initial response over time (LOR). ${ }^{25}$ Consequently, personalised medicine approaches need to be developed to avoid the risk of non-response to a drug, identify those patients most likely to benefit from specific therapies and choose the best treatment for each individual patient either at the initiation of the therapy or at the eventual LOR. ${ }^{26}$ Some studies conducted in other autoimmune diseases, such as rheumatoid arthritis, have shown the negative impact of the presence of comorbidities on therapeutic response to biologics. ${ }^{36}{ }^{37}$ In contrast, even though there have been a number of epidemiological studies assessing the prevalence of comorbidities associated with IBD ${ }^{14-16}{ }^{31}$ none has addressed the impact of the entire clinical profile of comorbidities and EIMs on the response to biologic therapy.

We found a statistically significant association among a few comorbidities and EIMs and both PNR to anti$\mathrm{TNF} \alpha$ during the induction phase (COPD) and LOR during the maintenance phase (myocardial infarction and skin diseases). As COPD, myocardial infarction and some skin diseases can aetiopathogenically be connected with smoking, it is plausible that the true factor behind the increased risk of PNR and LOR to anti-TNF $\alpha$ is 
Table 4 Multivariate logistic regression analysis of factors influencing efficacy

\begin{tabular}{|c|c|c|c|c|c|c|c|c|}
\hline & & & & & & & $95 \% \mathrm{C}$ & r OR \\
\hline & B & SE & Wald & df & $P$ value & OR & Lower & Upper \\
\hline Primary efficacy analysis: comorbidities as & with pr & ry non- & onse du & the & Iction pha & & & \\
\hline IBD & -0.54 & 0.24 & 5.09 & 1 & 0.024 & 0.59 & 0.37 & 0.93 \\
\hline Corticosteroids & 0.77 & 0.28 & 7.66 & 1 & 0.006 & 2.16 & 1.25 & 3.73 \\
\hline Chronic obstructive pulmonary disease & 0.98 & 0.35 & 7.70 & 1 & 0.006 & 2.67 & 1.33 & 5.35 \\
\hline Primary efficacy analysis: comorbidities as & with lo & f respc & during $t$ & hain & nce phase & & & \\
\hline IBD & -0.54 & 0.27 & 4.05 & 1 & 0.044 & 0.58 & 0.34 & 0.99 \\
\hline Corticosteroids & 0.90 & 0.30 & 8.67 & 1 & 0.003 & 2.45 & 1.35 & 4.44 \\
\hline Myocardial infarction & 1.20 & 0.41 & 8.57 & 1 & 0.003 & 3.30 & 1.48 & 7.35 \\
\hline Secondary efficacy analysis: EIMs associa & primary & 1-respc & during $t$ & duc & phase & & & \\
\hline Corticosteroids & 0.73 & 0.27 & 7.32 & 1 & 0.007 & 2.08 & 1.22 & 3.54 \\
\hline Constant & 0.83 & 0.40 & 4.21 & 1 & 0.04 & 2.29 & & \\
\hline Secondary efficacy analysis: EIMs associa & loss of & onse c & the $\mathrm{me}$ & nan & hase§ & & & \\
\hline Corticosteroids & 0.94 & 0.30 & 9.89 & 1 & 0.002 & 2.57 & 1.43 & 4.61 \\
\hline Skin disease & 1.01 & 0.33 & 9.13 & 1 & 0.003 & 2.73 & 1.42 & 5.25 \\
\hline
\end{tabular}

*Stepwise MLRA including as independent factors those comorbidities that were significant at $p<0.1$ in the univariate analysis, and as the dependent factor the primary non-response during the induction phase. In addition, type of IBD, treatment with immunosuppressants, and treatment with corticosteroids were included as fixed factors in the model.

†Stepwise MLRA including as independent factors those comorbidities that were significant at $p<0.1$ in the univariate analysis, and as the dependent factor the loss of response during the maintenance phase. In addition, type of IBD, treatment with immunosuppressants, and treatment with corticosteroids were included as fixed factors in the model.

¥Stepwise MLRA including as independent factors those EIMs that were significant at $p<0.1$ in the univariate analysis, and as the dependent factor the primary non-response during the induction phase. In addition, type of IBD, treatment with immunosuppressant, and treatment with corticosteroids were included as fixed factors in the model.

$\S$ Stepwise MLRA including as independent factors those EIMs that were significant at $p<0.1$ in the univariate analysis, and as the dependent factor the loss of response during the maintenance phase. In addition, type of IBD, treatment with immunosuppressants, and treatment with corticosteroids were included as fixed factors in the model.

EIMs, extraintestinal manifestations; IBD, inflammatory bowel disease; MLRA, multivariate logistic regression analysis.

smoking habit, in such a manner that COPD, myocardial infarction and skin diseases could be mere surrogates of smoking behaviour. However, this does not appear to be the case, as smoking habit was not identified under univariate analysis as a potential predictor of PNR ( $\mathrm{p}=0.670)$ or LOR $(\mathrm{p}=0.677)$. In any case, in order to test this hypothesis more in depth, additional multivariate analyses were conducted, forcing three different variables related with smoking behaviour as independent variables: current smoker, ex-smoker and ever smoker. Interestingly, whereas none of these three variables was identified as an independent predictor of PNR or LOR, COPD, myocardial infarction and skin diseases kept significance as independent predictors of PNR or LOR (data not shown). This reinforces that the determinants of failure to anti-TNF $\alpha$ therapy are the identified comorbidities/EIMs, rather than smoking habits.

These comorbidities/EIMs had increased prevalence in many epidemiological IBD studies, and some aetiopathogenic explanations have been hypothesised. ${ }^{38-40}$ Overexpression of inflammatory factors and abnormal immune responses have been postulated as the common pathogenetic mechanisms underlying IBD and comorbidities. In some epidemiological studies, a strong association between COPD and IBD was found. Compared with healthy controls, the risk of COPD in patients with CD was 2.7-fold higher, and in UC it was 1.8 -fold higher. ${ }^{38}$

In our study, COPD was among the most frequently reported comorbidities in both patients with $\mathrm{CD}(3.2 \%)$ and patients with UC (4.4\%). Likewise, acute myocardial infarction was found to be almost three times as likely in patients with IBD as in matched controls in a populationbased study. ${ }^{38}$ On the other hand, major skin involvement has also been described in $2 \%-34 \%$ of patients with IBD. ${ }^{39}$ However, it is difficult to diagnose these skin manifestations as EIMs because they can also arise as paradoxical reactions to anti-TNF $\alpha$ drugs. ${ }^{40}$ For example, some reports have suggested that inhibition of TNF $\alpha$ induces overexpression of cutaneous interferon- $\alpha$, which in turn causes a predisposition to psoriasis. ${ }^{40}$ Hence, in light of the present results, when selecting an anti-TNF $\alpha$ for the treatment of patients with IBD, the identified comorbidities and EIMs predicting a possible drug response failure should be taken into consideration.

The prevalence of comorbidities in our study was relatively low compared with other studies. ${ }^{14-16}$ A plausible explanation might be that the median age of study patients was relatively young (approximately 44 years old). Another reason could be that many IBD specialists or even patients are reluctant to use biologic 
therapy, and we cannot discard a possible overlap between comorbidities and EIMs due to the retrospective study design. We also found an unusual distribution of comorbidities in the subpopulations of patients with CD and UC. Unlike most of the epidemiological data, we found higher prevalence of comorbidities and EIMs in UC than in CD. Interestingly, patients with UC in our study showed a significant association with a more severe disease compared with patients with CD, and also showed a significant association with PNR or LOR compared with patients with CD. Therefore, the higher disease severity in patients with UC included in our study may explain the higher occurrence of EIMs in this group. These findings are nonetheless in line with other published results. ${ }^{41-43}$ Park et al found disease severity to be a strong predictor of non-response to infliximab in patients with $\mathrm{UC}^{43}{ }^{43}$ The authors hypothesised that, unlike CD, UC seems to result from an immune response of type 2 helper $\mathrm{T}$ cells in the colonic mucosa, suggesting that TNF $\alpha$ would play no important role in the pathogenesis of UC.

We also found a significant association between the use of corticosteroids and both PNR and LOR. Some published studies have reported the early use of corticosteroids as an independent predictor of a disabling disease ${ }^{44}$ of the need for anti-TNF $\alpha$ treatment dose intensification, and of an increased risk of anti-TNF $\alpha$ treatment failure. ${ }^{45}$ All these findings together appear to suggest that the early need for corticosteroids may be a proxy for disease severity or hard-to-treat disease.

Some of the limitations of the present study were the retrospective design and the lack of a control group. On the other hand, a requirement to a successful logistic regression model is to select appropriate variables to be entered into the model. While it is tempting to include as many input variables as possible, this can dilute true associations, or conversely identify spurious associations. In order to limit these risks, the conventional technique was followed, meaning to first run the univariate analyses to identify potential predictor variables, and then to use only those variables which meet a preset cut-off for significance to run a multivariate model.

Another limitation was the low frequency of comorbidities and EIMs observed, which may have reduced the statistical power to detect some other comorbidities or EIMs as predictors of PNR or LOR to anti-TNF $\alpha$ treatment in patients with IBD. In any case, the fact that some comorbidities or EIMs were identified as predictors of treatment failure, despite their low prevalence in the studied population, indicates that they show a strong association with PNR or LOR to anti-TNF $\alpha$ treatment. Furthermore, even though the UC patient sample was smaller than planned, which was probably because many patients with UC successfully responded to conventional therapies, both the final study sample size and the patient distribution were appropriate for meeting the study objectives.
Nonetheless, larger prospective controlled and preferably randomised studies are needed to confirm our results. In addition, a study assessing the impact of comorbidities on response to other biologics in the IBD population should be conducted to compare the study results with the results of the present study.

In conclusion, our results suggest that the presence of some comorbidities, such as COPD and myocardial infarction, and of certain EIMs of IBD, such as hepatopancreato-biliary conditions and skin diseases, seems to be connected to the lower probability of therapeutic success with anti-TNF $\alpha$. Therefore, the presence of these conditions in patients with IBD should guide clinicians when selecting the most appropriate treatment.

\section{Author affiliations}

${ }^{1}$ Department of Gastroenterology, Hospital General Universitario Gregorio Marañón, Madrid, Spain

${ }^{2}$ Instituto de Investigación Sanitaria Gregorio Marañón, Madrid, Spain ${ }^{3}$ Department of Gastroenterology, Hospital La Fe, Valencia, Spain

${ }^{4}$ Department of Gastroenterology, Hospital General de Castellón, Castellón, Spain ${ }^{5}$ Department of Gastroenterology, Hospital de la Santa Creu i Sant Pau, Barcelona, Spain

${ }^{6}$ Department of Gastroenterology, Hospital Universitario Virgen Macarena, Sevilla, Spain

${ }^{7}$ Medical Department, Takeda Farmacéutica España SA, Madrid, Spain

${ }^{8}$ Department of Gastroenterology, Hospital Universitario Río Hortega, Valladolid, Spain

${ }^{9}$ IBD Unit, Gastroenterology Department, University Clinic Hospital of Valencia, Valencia, Spain

${ }^{10}$ Department of Gastroenterology, Hospital Clínico Universitario de Santiago de Compostela, Santiago de Compostela, Spain

${ }^{11}$ Department of Gastroenterology, Hospital Universitario Cruces, Bilbao, Spain

${ }^{12}$ Department of Gastroenterology, Hospital Universitari de Girona Doctor Josep Trueta, Girona, Spain

${ }^{13}$ IBD Unit, Gastroenterology Section, Internal Medicine, Hospital de Sagunto, Sagunto, Spain

${ }^{14}$ Department of Gastroenterology, Hospital Universitario de Burgos, Burgos, Spain

${ }^{15}$ Department of Gastroenterology, Hospital Ramon y Cajal, Madrid, Spain

${ }^{16}$ Department of Gastroenterology, Hospital Clínico Universitario de Santiago de Compostela, Madrid, Spain

Acknowledgements We would like to thank Ana Sánchez-Gabriel (Takeda Farmacéutica España), Tamara Pozo (Takeda Farmacéutica España), Teresa Letosa (Takeda Farmacéutica España), Yaiza Gimeno (Takeda Farmacéutica España), Silvia Vives (Takeda Farmacéutica España), Susana Vara (Apices) and Juan Luis Sanz (Apices) for their support in design, set-up, coordination, operation, monitoring, statistical analysis and medical writing. The following investigators contributed to patient recruitment and data collection: Dra Montserrat Rivero Tirado (H Marqués de Valdecilla), Dr Oscar Roncero (H La Mancha Centro), Dra Yolanda Ber (H San Jorge), Dr Fernando Bermejo (H Fuenlabrada), Dra Cristina Verdejo (H Ciudad Real), Dra Cristina Rodríguez Gutierrez (H Navarra), Dra Belén Botella (H Infanta Cristina), Dra Pilar López Serrano (H Alcorcón), Dra Silvia Chacón (H Morales Meseguer), Dr Daniel Ceballos (H Dr Negrín) and Dra Pilar Varela (H Cabueñes).

Contributors All authors have contributed to and agreed on the content of the manuscript: IM-J, GB, AF, EG-P, FA-A, MA, JS-F, MMB-W, RF, OM, XA, XC, BS, $\mathrm{FM}$, and MB-A contributed to study design, patient recruitment, data collection and results interpretation. IT, AF-N, PS, and CM contributed to data analysis and interpretation, drafting of the report, and reviewed it critically. All authors approved the final version of the manuscript.

Funding The VERNE study was funded by Takeda Farmacéutica España SA.

Competing interests IM-J has served as a consultant, advisory member, speaker, or has received research funding from MSD, AbbVie, Takeda, Tillotts, Ferring, Falk Pharma, Faes Farma, UCB Pharma, Otsuka Pharmaceutical, Shire, Gebro Pharma, and Chiesi. GB has received a speaker honorarium from AbbVie, Pfizer, Janssen, FAES, Takeda, Tillotts and Abbott. Also, GB has participated in the 
scientific advisory committees of Takeda, Janssen and AbbVie. EG-P has served as a speaker or received research or educational funding or advisory fees from MSD, AbbVie, Janssen, Ferring, Shire, Tillotts, and FAES. FA-A has served as a speaker, a consultant and as an advisory member for or have received research funding from Janssen, MSD, AbbVie, Pfizer, Kern Pharma, Biogen, Sandoz, Takeda, Ferring, Faes Farma, Shire Pharmaceuticals, Dr Falk Pharma, Tillotts Pharma, Gebro Pharma, Amgen and Vifor Pharma. IT, AF-N, and CM are full employees of Takeda Farmacéutica España. PS was a Takeda Farmacéutica España employee at the moment this work was conducted. MA has served as a speaker for MSD, AbbVie Janssen, Takeda and Tillotts, and received educational grants from Janssen, MSD and AbbVie. JS-F has nothing to declare. MMB-W declares educational activities, research projects, scientific meetings and advisory boards sponsored by MSD, Ferring, AbbVie, Janssen and Takeda. RF has served as a speaker for or has received research funding from Takeda, MSD, AbbVie, Janssen, Palex, Shire Pharmaceuticals, Tillotts Pharma and Casen Recordati. OM has nothing to declare. MB-A has served as a speaker, a consultant and advisory board member for, or has received research funding from, MSD, AbbVie, Janssen, Pfizer, Kern Pharma, Biogen, Takeda, Ferring, Faes Farma, Shire Pharmaceuticals, Dr Falk Pharma, Tillotts Pharma, Chiesi, Gebro Pharma, Otsuka Pharmaceutical and Vifor Pharma.

\section{Patient consent for publication Not required.}

Ethics approval The study was reviewed and approved by the corresponding ethics committees.

\section{Provenance and peer review Not commissioned; externally peer reviewed.}

Data availability statement Data are available upon reasonable request. The data that support the findings of this study are available from the sponsor upon request.

Open access This is an open access article distributed in accordance with the Creative Commons Attribution Non Commercial (CC BY-NC 4.0) license, which permits others to distribute, remix, adapt, build upon this work non-commercially, and license their derivative works on different terms, provided the original work is properly cited, appropriate credit is given, any changes made indicated, and the use is non-commercial. See: http://creativecommons.org/licenses/by-nc/4.0/.

ORCID iD

Ignacio Marin-Jimenez http://orcid.org/0000-0001-5424-2484

\section{REFERENCES}

1 Podolsky DK. Inflammatory bowel disease. N Engl J Med 2002;347:417-29.

2 Baumgart DC, Sandborn WJ. Inflammatory bowel disease: clinical aspects and established and evolving therapies. Lancet 2007;369:1641-57.

3 Sin AT, Damman JL, Ziring DA, et al. Out-of-pocket cost burden in pediatric inflammatory bowel disease: a cross-sectional cohort analysis. Inflamm Bowel Dis 2015;21:1368-77.

4 Crumbock SC, Loeb SJ, Fick DM. Physical activity, stress, disease activity, and quality of life in adults with Crohn disease. Gastroenterol Nurs 2009;32:188-95

5 Irvine EJ. Quality of life of patients with ulcerative colitis: past, present, and future. Inflamm Bowel Dis 2008;14:554-65.

6 Büsch K, da Silva SA, Holton M, et al. Sick leave and disability pension in inflammatory bowel disease: a systematic review. $J$ Crohns Colitis 2014;8:1362-77.

7 Juan J, Estiarte R, Colomé E, et al. Burden of illness of Crohn's disease in Spain. Dig Liver Dis 2003;35:853-61.

8 Loftus EV. Clinical epidemiology of inflammatory bowel disease: incidence, prevalence, and environmental influences. Gastroenterology 2004;126:1504-17.

9 Shivananda S, Lennard-Jones J, Logan R, et al. Incidence of inflammatory bowel disease across Europe: is there a difference between North and South? results of the European collaborative study on inflammatory bowel disease (EC-IBD). Gut 1996;39:690-7.

10 Carpio D, Barreiro-de Acosta M, Echarri A, et al. Influence of urban/ rural and coastal/inland environment on the prevalence, phenotype, and clinical course of inflammatory bowel disease patients from northwest of Spain: a cross-sectional study. Eur J Gastroenterol Hepatol 2015;27:1030-7.

11 Pajares J, Gisbert J. Epidemiología de la enfermedad inflamatoria intestinal en España. Una revisión sistemática. Rev Esp Enferm Dig 2001;93:9-14.

12 Sicilia B, Vicente R, Gomollón F. Enfermedad de Crohn Y colitis ulcerosa: discusión de la epidemiología clásica. Acta Gastroenterol Latinoam 2009;39:135-45.
13 Román ALS, Muñoz F. Comorbidity in inflammatory bowel disease. World J Gastroenterol 2011;17:2723-33.

14 Bähler C, Schoepfer AM, Vavricka SR, et al. Chronic comorbidities associated with inflammatory bowel disease: prevalence and impact on healthcare costs in Switzerland. Eur J Gastroenterol Hepatol 2017;29:916-25.

15 Bernstein CN, Wajda A, Blanchard JF. The clustering of other chronic inflammatory diseases in inflammatory bowel disease: a populationbased study. Gastroenterology 2005;129:827-36.

16 Haapamäki J, Roine RP, Turunen U, et al. Increased risk for coronary heart disease, asthma, and connective tissue diseases in inflammatory bowel disease. J Crohns Colitis 2011;5:41-7.

17 Olpin JD, Sjoberg BP, Stilwill SE, et al. Beyond the bowel: extraintestinal manifestations of inflammatory bowel disease. Radiographics 2017;37:1135-60.

18 Harbord M, Annese V, Vavricka SR, et al. The first European evidence-based consensus on extra-intestinal manifestations in inflammatory bowel disease. J Crohns Colitis 2016;10:239-54.

19 Vavricka SR, Schoepfer A, Scharl M, et al. Extraintestinal manifestations of inflammatory bowel disease. Inflamm Bowel Dis 2015;21:1982-92.

20 Renna S, Cottone M, Orlando A. Optimization of the treatment with immunosuppressants and biologics in inflammatory bowel disease. World J Gastroenterol 2014;20:9675-90.

21 Baert F, Moortgat L, Van Assche G, et al. Mucosal healing predicts sustained clinical remission in patients with early-stage Crohn's disease. Gastroenterology 2010;138:463-8.

22 Zallot C, Peyrin-Biroulet L. Deep remission in inflammatory bowel disease: looking beyond symptoms. Curr Gastroenterol Rep 2013;15:315.

23 Ordás I, Feagan BG, Sandborn WJ. Early use of immunosuppressives or TNF antagonists for the treatment of Crohn's disease: time for a change. Gut 2011;60:1754-63.

24 Ford AC, Sandborn WJ, Khan KJ, et al. Efficacy of biological therapies in inflammatory bowel disease: systematic review and meta-analysis. Am J Gastroenterol 2011;106:644-59.

25 Martínez-Montiel MP, Casis-Herce B, Gómez-Gómez GJ, et al. Pharmacologic therapy for inflammatory bowel disease refractory to steroids. Clin Exp Gastroenterol 2015;8:257-69.

26 Allez M, Karmiris K, Louis E, et al. Report of the ECCO pathogenesis workshop on anti-TNF therapy failures in inflammatory bowel diseases: definitions, frequency and pharmacological aspects. J Crohns Colitis 2010;4:355-66.

27 Ben-Horin S, Chowers Y. Review article: loss of response to anti-TNF treatments in Crohn's disease. Aliment Pharmacol Ther 2011;33:987-95

28 Naviglio S, Giuffrida P, Stocco G, et al. How to predict response to anti-tumour necrosis factor agents in inflammatory bowel disease. Expert Rev Gastroenterol Hepatol 2018;12:797-810.

29 Atreya R, Neurath MF. Mechanisms of molecular resistance and predictors of response to biological therapy in inflammatory bowel disease. Lancet Gastroenterol Hepatol 2018;3:790-802.

30 Lopetuso LR, Gerardi V, Papa V, et al. Can we predict the efficacy of anti-TNF- $\alpha$ agents? Int J Mol Sci 2017;18:E1973.

31 Bernstein CN, Fried M, Krabshuis JH, et al. World gastroenterology organization practice guidelines for the diagnosis and management of IBD in 2010. Inflamm Bowel Dis 2010;16:112-24.

32 Lewis JD, Chuai S, Nessel L, et al. Use of the noninvasive components of the Mayo score to assess clinical response in ulcerative colitis. Inflamm Bowel Dis 2008;14:1660-6.

33 Harvey RF, Bradshaw JM. A simple index of Crohn's-disease activity. Lancet 1980;1:514

34 Lobatón T, Ferrante M, Rutgeerts P, et al. Efficacy and safety of antiTNF therapy in elderly patients with inflammatory bowel disease. Aliment Pharmacol Ther 2015;42:441-51.

35 Charlson ME, Pompei P, Ales KL, et al. A new method of classifying prognostic comorbidity in longitudinal studies: development and validation. J Chronic Dis 1987;40:373-83.

36 Armagan B, Sari A, Erden A, et al. Starting of biological disease modifying antirheumatic drugs may be postponed in rheumatoid arthritis patients with multimorbidity: single center real life results. Medicine 2018;97:e9930.

37 Krishnan E, Häkkinen A, Sokka T, et al. Impact of age and comorbidities on the criteria for remission and response in rheumatoid arthritis. Ann Rheum Dis 2005;64:1350-2.

38 Hornikx M, Van Remoortel H, Demeyer H, et al. The influence of comorbidities on outcomes of pulmonary rehabilitation programs in patients with COPD: a systematic review. Biomed Res Int 2013:2013:146148.

39 Vutcovici M, Brassard P, Bitton A. Inflammatory bowel disease and airway diseases. World J Gastroenterol 2016;22:7735-41. 
40 Aniwan S, Pardi DS, Tremaine WJ, et al. Increased risk of acute myocardial infarction and heart failure in patients with inflammatory bowel diseases. Clin Gastroenterol Hepatol 2018;16:1607-15.

41 Huang BL, Chandra S, Shih DQ. Skin manifestations of inflammatory bowel disease. Front Physiol 2012;3:13.

42 Zippi M, Pica R, De Nitto D, et al. Biological therapy for dermatological manifestations of inflammatory bowel disease. World J Clin Cases 2013;1:74-8.
43 Park SH, Yang S-K, Hong S-M, et al. Severe disease activity and cytomegalovirus colitis are predictive of a nonresponse to infliximab in patients with ulcerative colitis. Dig Dis Sci 2013;58:3592-9.

44 Seow CH, Newman A, Irwin SP, et al. Trough serum infliximab: a predictive factor of clinical outcome for infliximab treatment in acute ulcerative colitis. Gut 2010;59:49-54

45 Oussalah A, Evesque L, Laharie D, et al. A multicenter experience with infliximab for ulcerative colitis: outcomes and predictors of response, optimization, colectomy, and hospitalization. Am J Gastroenterol 2010;105:2617-25. 